Research Article

\title{
Elastic Wave Properties of an Adaptive Electromechanical Metamaterial Beam
}

\author{
Xi-Ning Zhao and Xiao-Dong Yang $\mathbb{B}$ \\ Beijing Key Laboratory of Nonlinear Vibrations and Strength of Mechanical Structures, College of Mechanical Engineering, \\ Beijing University of Technology, Beijing 100124, China \\ Correspondence should be addressed to Xiao-Dong Yang; jxdyang@163.com
}

Received 13 July 2020; Revised 15 September 2020; Accepted 6 October 2020; Published 21 October 2020

Academic Editor: Giuseppe Brandonisio

Copyright (c) $2020 \mathrm{Xi-Ning} \mathrm{Zhao} \mathrm{and} \mathrm{Xiao-Dong} \mathrm{Yang.} \mathrm{This} \mathrm{is} \mathrm{an} \mathrm{open} \mathrm{access} \mathrm{article} \mathrm{distributed} \mathrm{under} \mathrm{the} \mathrm{Creative} \mathrm{Commons}$ Attribution License, which permits unrestricted use, distribution, and reproduction in any medium, provided the original work is properly cited.

\begin{abstract}
An adaptive metamaterial beam with a couple of electromechanical resonators in each unit cell is proposed in this study to open Bragg bandgap or locally resonant bandgap for flexural wave attenuation in Euler-Bernoulli beams. The electromechanical resonator is composed of a piezoelectric layer with segmented electrodes and shunt circuits, which affect the dynamic equivalent stiffness. It is illustrated that there is only a Bragg bandgap when the circuits of the two adjacent resonators are approximate to a short circuit or open circuit, and the locally resonant bandgap will be generated in the pure inductance circuits when the resonant frequencies are different in general. The locally resonant bandgap can be broadened by adding more resonators into the unit cell with the resonant frequencies of the shunting circuits satisfying a proper ratio.
\end{abstract}

\section{Introduction}

The research of acoustic/elastic metamaterials has lately attracted growing attention [1-3]. In particular, locally resonant metamaterials yield bandgap formation at wavelengths much larger than the lattice size of the substructure, and acoustic metamaterial has shown promising advantages in enabling vibration absorption and sound attenuation. In 2000, Liu et al. firstly used hard metal shperes embedded in a soft rubber as mechanical resonators to demonstrate the locally resonant bandgap properties. This seminal work opened up a new way for the study of acoustic metamaterials.

A variety of metamaterials with purely mechanical locally resonators have been researched using the plane wave expansion method, transfer matrix method, or finite element method [5-10]. However, the resonant bandwidth yielded by elastic/mechanical metamaterials is narrow, which seriously limits their use in noise or vibration absorption. Moreover, actively controlling the position and width of the bandgap frequency region is very difficult in practice, if not impossible, for passive metamaterials.
One of the most pronounced challenges in elastic metamaterial development is the ability to tune their performance in an adaptive manner without requiring physical structural modifications. The piezoelectric shunting technique was originally proposed by Forward [11] and further investigated by Hagood and von Flotow [12]. These electromechanical resonators have the benefit of significantly lower mass requirements than purely mechanical massspring resonators. The use of piezoelectric elements shunted to resonating circuits has lately been explored for locally resonant bandgap formation. These structures with resonating circuits can exhibit an electromechanical locally resonant bandgap.

Recently, Sugino et al. $[13,14]$ derived the bound frequencies of the locally resonant bandgap for a piezoelectric bimorph beam, finding that the purely piezoelectric locally resonant bandgap size mainly depends on the electromechanical coupling coefficient. Xu et al. [15] established a semianalytical model of adaptive piezoelectric materials and proposed a new method to widen the bandgap by adding geometric cavities, which can increase the electromechanical coupling coefficient and effectively broaden the bandwidth. 
A one-dimensional piezoelectric stack phononic crystal with a shunt circuit was studied by Chen et al. [16] and Lia et al. [17] using the transfer matrix method. Theoretical and numerical results show that the shunt circuits can tune the regulation of structure flexural wave propagation. In addition, a hybrid adaptive metamaterial $[18,19]$ with mechanical and electromechanical resonators is proposed to control the flexural waves and reveals that the phenomena for the mechanical and electromechanical locally resonant bandgaps are caused by the negative dynamic mass and negative dynamic stiffness, respectively. Two types of locally resonant bandgaps can be combined into a single, larger bandgap for increased bandwidth, by adjusting the natural frequency of the mechanical and electromechanical resonators.

In order to be suitable for more structural applications, two-dimensional electromechanical metamaterials with segmented electrodes and specified boundary conditions have been studied. Recently, to control the wave propagation in a thin plate, two-dimensional metamaterials with periodic arrays of shunted piezoelectric patches have been employed in Zhang et al. [20] by using the finite element method and Bloch theorem.

Kherraz et al. [21] demonstrated numerically and experimentally the opening of a locally resonant bandgap in an active phononic crystal and the application of periodic electroboundary conditions to control the dispersion of elastic waves in plates. By using Hamilton's principle, Sugino et al. [22] established a fully coupled two-dimensional governing equation and boundary conditions of the piezoelectric plate and found that the effective dynamic stiffness of the system depends on the admittance of the shunt circuit and electromechanical coupling in the system. By using the plane wave expansion method and the modal expansion method, the local resonant bandgap and wave attenuation have been obtained, and the finite element method was used to verify the analytical results. Metamaterials with piezoelectric shunting technology can also be used for active vibration control $[23,24]$ and piezoelectric energy harvesting [25-28].

Inspired by Xiao et al. [29] and Zhou et al. [10], more mechanical resonators are added to the unit cell to broaden the bandgaps, especially for very low target frequencies. In this paper, a couple of electromechanical resonators containing a piezoelectric layer with segmented electrodes and shunt circuit in one-unit cell are proposed to open Bragg bandgap or locally resonant bandgap for flexural wave attenuation in Euler-Bernoulli beams. Band structures in both pure resistance and inductance circuits have been studied by using the transfer matrix method and the discretization method will be applied as numerical validation. The effects of the circuit parameters, connections, and length ratio on the band structures of this electromechanical metamaterial beam are investigated. The analytical and numerical results demonstrate that a couple of electromechanical resonators in each unit cell is practicable to open Bragg bandgap or locally resonant bandgap. Our design is of great significance for the practical applications of low-frequency vibration or wave attenuations in engineering.

\section{Model and Method of Calculation}

2.1. Structure Model. Figure 1(a) shows a simplified structural modal of our designed adaptive metamaterial beam, which is comprised of a host beam with periodically surfacebonded piezoelectric layers. Each of the piezoelectric layers with opposite polarizations in the transverse direction is made of two rectangular cross-section piezoelectric plates. The structure of each unit cell containing two subcells named subcell 1 and subcell 2 is illustrated in Figure 1(b). The symbols $l_{1}$ and $l_{2}$ represent the length of subcell 1 and subcell 2, respectively, $h_{\mathrm{s}}$ and $h_{\mathrm{p}}$ are the thicknesses of the host beam and piezoelectric layers, and $v_{j 1}, v_{j 2}$ and $Y_{j 1}, Y_{j 2}$ are the voltage and the external load admittance across the two subcells of the $j$ th electrode pair. The host beam and the piezoelectric layer share the same width $b$. The circuits of the subcells 1 and 2 have the same connections and different impedances, which causes different dynamic effective modulus and different natural frequencies. It will be found that the periodic arranged piezoelectric layers and alternate impedances give rise to various phenomena of band structures.

In the following two subsections, the transfer matrix method and modes discretization method are used, respectively, to infinite and finite modes to validate the results of each other.

\subsection{Propagation of Flexural Waves in Electromechanical} Metamaterial Beam by Using the Transfer Matrix Method. The mechanical interface boundary effect between the host beam and the piezoelectric layers is ignored since the bonding is assumed to be perfect. The constitutive equations of the piezoelectric beam are

$$
\begin{aligned}
& S=s_{11}^{E} T+d_{31} E_{3}, \\
& D=d_{31} T+\varepsilon_{33}^{T} E_{3},
\end{aligned}
$$

where $S$ and $T$ are the normal stress and normal strain in the $x$-direction and $D$ and $E_{3}$ denote the electric displacement and electric field in $z$-direction, respectively. The symbol $s_{11}^{E}$ is the elastic compliance at the constant electric field, $d_{31}$ is the piezoelectric strain constant, and $\varepsilon_{33}^{T}$ is the permittivity component at constant stress. Here, for the modeling of the piezoelectric metamaterial, we adopt the equivalent Young's modulus representation [12]

$$
\begin{aligned}
& E_{\mathrm{p}, j 1}=E_{\mathrm{p}}\left(1-\frac{k_{31}^{2}}{1+i \omega C_{\mathrm{p}, j 1} Z_{j 1}(\omega)}\right), \\
& E_{\mathrm{p}, j 2}=E_{\mathrm{p}}\left(1-\frac{k_{31}^{2}}{1+i \omega C_{\mathrm{p}, j 2} Z_{j 2}(\omega)}\right),
\end{aligned}
$$

where $Z_{j 1}(\omega)$ and $Z_{j 2}(\omega)$ are the impedance of the two subcells, depending on the type of the shunt circuit and the value of the circuit element. The impedance $Z_{j}$ and the admittance $Y_{j}$ mentioned in Figure 1 are reciprocal of each other $Y_{j}=1 / Z_{j}$. In pure resistance/inductance circuit, the 


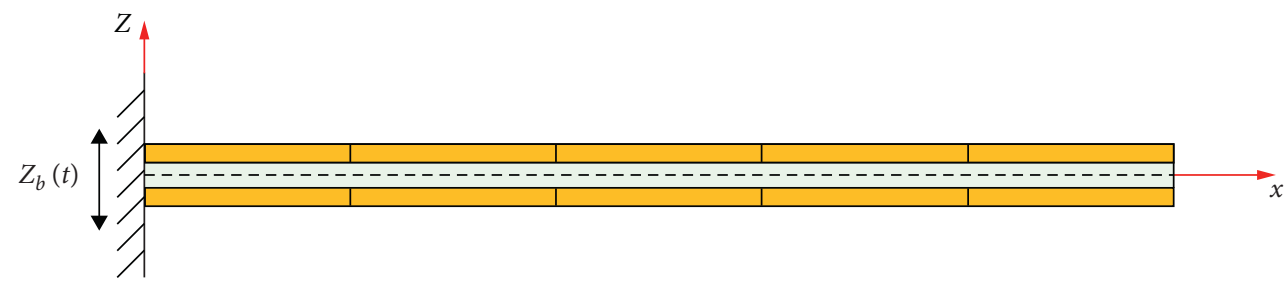

(a)

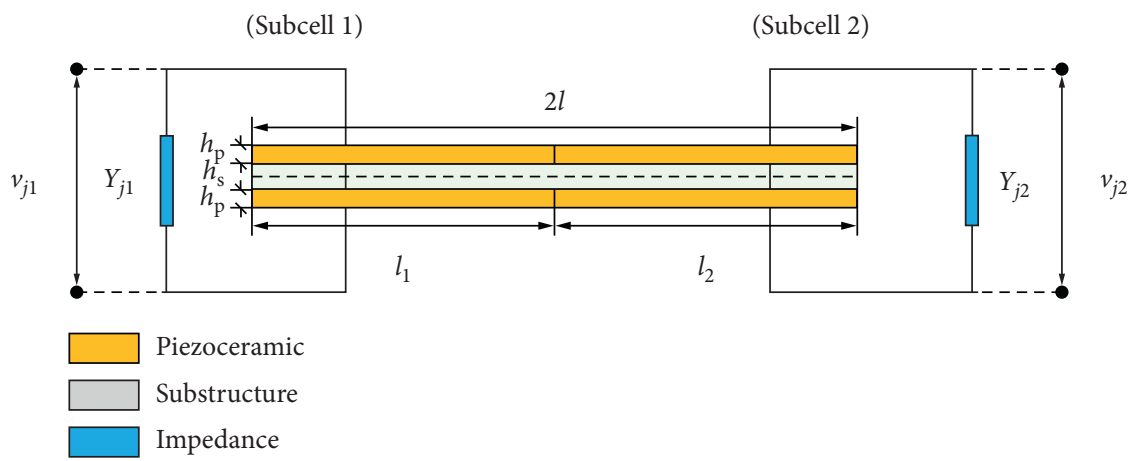

(b)

FIGURE 1: Simplified structural modal: (a) locally resonant metamaterials beam and (b) unit cell.

impedance can be expressed as $R_{j} / i \omega L_{j}$. The circuit can be approximate to open/short circuit when the impedance is infinite or goes to zero indefinitely. The short-circuit Young's modulus $E_{\mathrm{p}}$, electromechanical coupling coefficient $k_{31}$, the permittivity component at constant strain $\varepsilon_{33}^{S}$, and the capacitance of the subcell 1 and subcell $2 C_{p, j 1}$ and $2 C_{p, j 2}$ are, respectively,

$$
\begin{gathered}
E_{\mathrm{p}}=\frac{1}{s_{11}^{E}}, \\
k_{31}=\sqrt{\frac{d_{31}^{2}}{s_{11}^{E} \varepsilon_{33}^{T}},} \\
\varepsilon_{33}^{S}=\varepsilon_{33}^{T}-\frac{d_{31}^{2}}{s_{11}^{E}}, \\
C_{\mathrm{p}, j 1}=\varepsilon_{33}^{S} b \frac{l_{1}}{2 h_{\mathrm{p}}}, \\
C_{\mathrm{p}, j 2}=\varepsilon_{33}^{S} b \frac{l_{2}}{2 h_{\mathrm{p}}} \sqrt{2} .
\end{gathered}
$$

In order to study the complex band structure of electromechanical metamaterial beams, the traditional transfer matrix method is used. Based on the Euler-Bernoulli beam theory, the governing equation of the transverse displacement for the host beam segment can be written as

$$
\mathrm{EI} \frac{\partial^{4} w(x, t)}{\partial x^{4}}+m \frac{\partial^{2} w(x, t)}{\partial t^{2}}=0
$$

where

$$
\begin{gathered}
\mathrm{EI}= \begin{cases}E_{\mathrm{s}} I_{\mathrm{s}}+E_{\mathrm{p}, j 1} I_{\mathrm{p}}, & 0 \leq x<l_{1}, \\
E_{\mathrm{s}} I_{\mathrm{s}}+E_{\mathrm{p}, j 2} I_{\mathrm{p}}, & l_{1} \leq x<2 l,\end{cases} \\
m= \begin{cases}\rho_{\mathrm{s}} A_{\mathrm{s}}+2 \rho_{\mathrm{p}} A_{\mathrm{p}}, & 0 \leq x<l_{1}, \\
\rho_{\mathrm{s}} A_{\mathrm{s}}+2 \rho_{\mathrm{p}} A_{\mathrm{p}}, & l_{1} \leq x<2 l .\end{cases}
\end{gathered}
$$

In equations (5)-(7), $w(x, t)$ denotes the transverse displacement of the host beam at position $x$ and time $t$. Symbols $E_{s}, \rho_{s}, I_{s}$, and $A_{s}$ are Young's modulus, mass density, area moment of inertia, and area of cross section of the host beam, respectively. Symbols $E_{\mathrm{p}}, \rho_{\mathrm{p}}, I_{\mathrm{p}}$, and $A_{\mathrm{p}}$ are Young's modulus under constant electric field, mass density, area moment of inertia, and area of cross-section of the piezoelectric plate, respectively.

To characterize the wave-attenuation properties of the metamaterial beam, the harmonic solution to equation (5) can be written as

$$
w(x, t)=W(x) e^{i \omega t},
$$

where $W(x)$ is the mode shape function and $\omega$ is the frequency of vibration. The general solution of subcell 1 and subcell 2 for the $j$ th unit cell can be written as

$$
\begin{aligned}
W_{j 1}(x)= & A_{j 1} \cos \quad k_{1} x+B_{j 1} \sin \quad k_{1} x \\
& +C_{j 1} \cosh \quad k_{1} x+D_{j 1} \sinh \quad k_{1} x \\
W_{j 2}(x)= & A_{j 2} \cos \quad k_{2}\left(x-l_{1}\right)+B_{j 2} \sin \quad k_{2}\left(x-l_{1}\right) \\
& +C_{j 2} \cosh \quad k_{2}\left(x-l_{1}\right)+D_{j 2} \sinh \quad k_{2}\left(x-l_{1}\right),
\end{aligned}
$$

where $A_{j}, B_{j}, C_{j}$, and $D_{j}$ are unknown constant and 


$$
\begin{aligned}
& k_{1}=\sqrt[4]{\omega^{2} \frac{\rho_{\mathrm{s}} A_{\mathrm{s}}+2 \rho_{\mathrm{p}} A_{\mathrm{p}}}{E_{\mathrm{s}} I_{\mathrm{s}}+E_{\mathrm{p}, j 1} I_{\mathrm{p}}}}, \\
& k_{2}=\sqrt[4]{\omega \frac{{ }^{2} \rho_{\mathrm{s}} A_{\mathrm{s}}+2 \rho_{\mathrm{p}} A_{\mathrm{p}}}{E_{\mathrm{s}} I_{\mathrm{s}}+E_{\mathrm{p}, j 2} I_{\mathrm{p}}}} .
\end{aligned}
$$

Consider the continuity conditions of the displacement, rotation angle, bending moment, and shear force at the connecting point between the two subcells:

$$
\begin{aligned}
W_{j 1}\left(l_{1}\right) & =W_{j, 2}(0), \\
W_{j 1}^{\prime}\left(l_{1}\right) & =W_{j, 2}^{\prime}(0), \\
\left(E_{\mathrm{s}} I_{\mathrm{s}}+E_{\mathrm{p}, j 1} I_{\mathrm{p}}\right) W_{j 1}^{\prime \prime}\left(l_{1}\right) & =\left(E_{\mathrm{s}} I_{\mathrm{s}}+E_{\mathrm{p}, j 2} I_{\mathrm{p}}\right) W_{j 2}^{\prime \prime}(0), \\
\left(E_{\mathrm{s}} I_{\mathrm{s}}+E_{\mathrm{p}, j 1} I_{\mathrm{p}}\right) W_{j 1}^{\prime \prime \prime}\left(l_{1}\right) & =\left(E_{\mathrm{s}} I_{\mathrm{s}}+E_{\mathrm{p}, j 2} I_{\mathrm{p}}\right) W_{j 2}^{\prime \prime \prime}(0) .
\end{aligned}
$$

By substituting equations (9) and (10) into equation (12), we can express the continuity conditions in the matrix form as

$$
\mathbf{H}_{1} \psi_{j 1}=\mathbf{K}_{2} \psi_{j 2},
$$

$$
\begin{aligned}
& \psi_{j 1}=\left\{\begin{array}{llll}
A_{j 1} & B_{j 1} & C_{j 1} & D_{j 1}
\end{array}\right\}^{T}, \\
& \psi_{j 2}=\left\{\begin{array}{llll}
A_{j 2} & B_{j 2} & C_{j 2} & D_{j 2}
\end{array}\right\}^{T},
\end{aligned}
$$

and

where

$$
\begin{aligned}
\mathbf{H}_{1} & =\left[\begin{array}{cccccccc}
\cos & k_{1} l_{1} & \sin & k_{1} l_{1} & \cosh & k_{1} l_{1} & \sinh & k_{1} l_{1} \\
-k_{1} \sin & k_{1} l_{1} & k_{1} \cos & k_{1} l_{1} & k_{1} \sinh & k_{1} l_{1} & k_{1} \cosh & k_{1} l_{1} \\
-E_{1} I k_{1}^{2} \cos & k_{1} l_{1} & -E_{1} I k_{1}^{2} \sin & k_{1} l_{1} & E_{1} I k_{1}^{2} \cosh & k_{1} l_{1} & E_{1} I k_{1}^{2} \sinh & k_{1} l_{1} \\
E_{1} I k_{1}^{3} \sin & k_{1} l_{1} & -E_{1} I k_{1}^{3} \cos & k_{1} l_{1} & E_{1} I k_{1}^{3} \sinh & k_{1} l_{1} & E_{1} I k_{1}^{3} \cosh & k_{1} l_{1}
\end{array}\right], \\
\mathbf{K}_{2} & =\left[\begin{array}{ccccc}
1 & 0 & 1 & 0 \\
0 & k_{2} & 0 & k_{2} \\
-E_{2} I k_{2}^{2} & 0 & E_{2} I k_{2}^{2} & 0 \\
0 & -E_{2} I k_{2}^{3} & 0 & E_{2} I k_{2}^{3}
\end{array}\right] .
\end{aligned}
$$

In equations (15) and (16),

$$
\begin{aligned}
& E_{1} I=E_{\mathrm{s}} I_{\mathrm{s}}+E_{\mathrm{p}, j 1} I_{\mathrm{p}}, \\
& E_{2} I=E_{\mathrm{s}} I_{\mathrm{s}}+E_{\mathrm{p}, j 2} I_{\mathrm{p}} .
\end{aligned}
$$

Similarly, applying the continuity conditions of the displacement, rotation angle, bending moment, and shear force between the two consecutive unit cells, we have

$$
\begin{aligned}
W_{j 2}\left(l_{2}\right) & =W_{(j+1) 1}(0), \\
W_{j 2}^{\prime}\left(l_{2}\right) & =W_{(j+1) 1}^{\prime}(0), \\
\left(E_{\mathrm{s}} I_{\mathrm{s}}+E_{\mathrm{p}, j 2} I_{\mathrm{p}}\right) W_{j, 2}^{\prime \prime}\left(l_{2}\right) & =\left(E_{\mathrm{s}} I_{\mathrm{s}}+E_{\mathrm{p},(j+1) 1} I_{\mathrm{p}}\right) W_{(j+1) 1}^{\prime \prime}(0), \\
\left(E_{\mathrm{s}} I_{\mathrm{s}}+E_{\mathrm{p}, j 2} I_{\mathrm{p}}\right) W_{j, 2}^{\prime \prime \prime}\left(l_{2}\right) & =\left(E_{\mathrm{s}} I_{\mathrm{s}}+E_{\mathrm{p},(j+1) 1} I_{\mathrm{p}}\right) W_{(j+1) 1}^{\prime \prime \prime}(0) .
\end{aligned}
$$

Each unit has the same material structure parameters and circuit parameters and the equivalent Young's modulus $E_{\mathrm{p}, j 1}=E_{\mathrm{p},(j+1) 1}$ and $E_{\mathrm{p}, j 2}=E_{\mathrm{p},(j+1) 2}$. By substituting equations
(9) and (10) into equation (19), we express the continuity conditions in the matrix form as

$$
\mathbf{H}_{2} \psi_{j 2}=\mathbf{K}_{1} \psi_{(j+1) 1},
$$


where

$$
\begin{aligned}
& \psi_{j 2}=\left\{\begin{array}{llll}
A_{j 2} & B_{j 2} & C_{j 2} & D_{j 2}
\end{array}\right\}^{T}, \\
& \Psi_{(j+1) 1}=\left\{\begin{array}{llll}
A_{(j+1) 1} & B_{(j+1) 1} & C_{(j+1) 1} & D_{(j+1) 1}
\end{array}\right\}^{T}, \\
& \mathbf{H}_{2}=\left[\begin{array}{cccccccc}
\cos & k_{2} l_{2} & \sin & k_{2} l_{2} & \cosh & k_{2} l_{2} & \sinh & k_{2} l_{2} \\
-k_{2} \sin & k_{2} l_{2} & k_{2} \cos & k_{2} l_{2} & k_{2} \sinh & k_{2} l_{2} & k_{2} \cosh & k_{2} l_{2} \\
-E_{2} I k_{2}^{2} \cos & k_{2} l_{2} & -E_{2} I k_{2}^{2} \sin & k_{2} l_{2} & E_{2} I k_{2}^{2} \cosh & k_{2} l_{2} & E_{2} I k_{2}^{2} \sinh & k_{2} l_{2} \\
E_{2} I k_{2}^{3} \sin & k_{2} l_{2} & -E_{2} I k_{2}^{3} \cos & k_{2} l_{2} & E_{2} I k_{2}^{3} \sinh & k_{2} l_{2} & E_{2} I k_{2}^{3} \cosh & k_{2} l_{2}
\end{array}\right] \text {, } \\
& \mathbf{K}_{1}=\left[\begin{array}{cccc}
1 & 0 & 1 & 0 \\
0 & k_{1} & 0 & k_{1} \\
-E_{1} I k_{1}^{2} & 0 & E_{1} I k_{1}^{2} & 0 \\
0 & -E_{1} I k_{1}^{3} & 0 & E_{1} I k_{1}^{3}
\end{array}\right] \text {. }
\end{aligned}
$$
yield

Combining equations (13) and (20) and eliminating $\psi_{j 2}$

$$
\boldsymbol{\psi}_{(j+1) 1}=\left(\mathbf{K}_{1}^{-1} \mathbf{H}_{2} \mathbf{K}_{2}^{-1} \mathbf{H}_{1}\right) \boldsymbol{\psi}_{j 1} .
$$

The transfer matrix between the two cells is then defined as

$$
\mathbf{T}=\mathbf{K}_{1}^{-1} \mathbf{H}_{2} \mathbf{K}_{2}^{-1} \mathbf{H}_{1} .
$$

Further, by employing the Floquet-Bloch theorem, for a one-dimensional periodic structure, the relationship between $\psi_{j+1}$ and $\psi_{j}$ is

$$
\psi_{(j+1) 1}=e^{\mathrm{i} q 2 l} \psi_{j 1},
$$

where $\mathbf{q}$ is the wave vector along the $x$-direction. By inserting equation (24) into equation (22), a standard eigenvalue problem is formed

$$
\left|\mathbf{T}-e^{\mathrm{i} q 2 l} \mathbf{I}\right|=0,
$$

where $\mathbf{I}$ is the $4 \times 4$ identity matrix. The dispersion relations of the metamaterial beam with electromechanical resonators can be obtained by solving the above dispersion equation (25). It can be seen that the dispersion equation is related to the equivalent Young's modulus mentioned in equations (2) and (3), which is affected by the circuit elements, including resistance, inductance, and inherent capacitance. The effects of short circuit and open circuit will be taken into account in further discussions. Based on the given frequencies, the complex wavenumbers are determined, from which the wave-propagation properties are derived. The propagation of a flexural wave in the beam is shown bandgap or passband when the solution of $\mathbf{q}$ is imaginary or real, correspondingly.

\subsection{Modes Discretization of the Electromechanical Meta-} material Beam with a Finite Length. For the purpose of the practical design, it is necessary to investigate the frequency response of the metamaterial beam with a finite number of electromechanical resonators. A uniform Euler-Bernoulli beam with piezoelectric layers is considered. The metamaterial beam with transverse base excitation $w_{b}(t)$ is of length $L$, and $2 S$ electromechanical resonators are periodically placed onto the host beam at the distance of $l_{1}$ and $l_{2}$ and $l_{1}$ and $l_{2}$ satisfying $l_{1}+l_{2}=2 l$. Without loss of generality, we assume the host beam and the resonators are all undamped and the length of the two subcells is the same, $l_{1}=l_{2}=l$. The governing equation for the electromechanical metamaterial beam is then

$$
\begin{gathered}
\text { EI } \frac{\partial^{4} w}{\partial x^{4}}+m \frac{\partial^{2} w}{\partial t^{2}}-\vartheta \sum_{j=1}^{S} v_{j 1} \frac{\mathrm{d}^{2}}{\mathrm{~d} x^{2}}\left[H\left(x-x_{j-1}^{2}\right)-H\left(x-x_{j}^{1}\right)\right] \\
-\vartheta \sum_{j=1}^{S} v_{j 2} \frac{\mathrm{d}^{2}}{\mathrm{~d} x^{2}}\left[H\left(x-x_{j}^{1}\right)-H\left(x-x_{j}^{2}\right)\right]=f(x, t) .
\end{gathered}
$$

The governing equations for the shunt circuit of the two subcells are

$$
\begin{gathered}
C_{\mathrm{p}, j 1} \frac{\mathrm{d} v_{j 1}}{\mathrm{~d} t}+Y_{j 1} v_{j 1}+\vartheta \int_{x_{j-1}^{2}}^{x_{j}^{1}} \frac{\partial^{3} w}{\partial x^{2} \partial t} \mathrm{~d} x=0, \\
C_{\mathrm{p}, j 2} \frac{\mathrm{d} v_{j 2}}{\mathrm{~d} t}+Y_{j 2} v_{j 2}+\vartheta \int_{x_{j}^{1}}^{x_{j}^{2}} \frac{\partial^{3} w}{\partial x^{2} \partial t} \mathrm{~d} x=0,
\end{gathered}
$$

where $w(x, t)$ is the transverse displacement of the beam at position $x$ and time $t$ and $v_{j 1}$ and $Y_{j 1}$ are, respectively, the voltage and the external load admittance across the subcell 1 of the $j$ th electrode pair. $v_{j 2}$ and $Y_{j 2}$ are parameters of subcell 2. $H(x)$ is the Heaviside function, and $f(x, t)$ is the external forcing. Furthermore, EI and $m$ are the short-circuit flexural rigidity and mass per length of the beam motioned in equation (1), $\vartheta$ is the term associated with electromechanical coupling coefficient, and $C_{\mathrm{p}, j 1}$ and $C_{\mathrm{p}, j 2}$ are the internal piezoelectric capacitance across the subcell 1 and subcell 2 of $j$ th electrode pair, given by 


$$
\begin{aligned}
\mathrm{EI} & =\frac{2 b}{3}\left[E_{\mathrm{s}} \frac{h_{\mathrm{s}}^{3}}{8}+E_{\mathrm{p}}\left[\left(h_{\mathrm{p}}+\frac{h_{\mathrm{s}}}{2}\right)^{3}-\frac{h_{\mathrm{s}}^{3}}{8}\right]\right], \\
m & =b\left(\rho_{\mathrm{s}} h_{\mathrm{s}}+2 \rho_{\mathrm{p}} h_{\mathrm{p}}\right), \\
\vartheta & =\frac{\bar{e}_{31} b}{2 h_{\mathrm{p}}}\left[\left(h_{\mathrm{p}}+\frac{h_{\mathrm{s}}}{2}\right)^{2}-\frac{h_{\mathrm{s}}^{2}}{4}\right], \\
C_{\mathrm{p}, j 1} & =\frac{\varepsilon_{33}^{s} b\left(x_{j}^{1}-x_{j-1}^{2}\right)}{2 h_{\mathrm{p}}}, \\
C_{\mathrm{p}, j 2} & =\frac{\varepsilon_{33}^{s} b\left(x_{j}^{2}-x_{j}^{1}\right)}{2 h_{\mathrm{p}}} .
\end{aligned}
$$

We assume a modal expansion [30] for the transverse displacement of the beam $w(x, t)$

$$
w(x, t)=\sum_{r=1}^{N} \phi_{r}(x) \eta_{r}(t),
$$

where functions $\phi_{r}(x)$ are the normalized mode shape functions of the plain beam (i.e., without resonators) at short circuit, $\eta_{r}(t)$ are the generalized coordinates to be confirmed, and $N$ is the discretized number. The modal functions of the host beam satisfy the orthogonality conditions

$$
\begin{gathered}
\int_{0}^{L} m \phi_{r}(x) \phi_{k}(x) \mathrm{d} x=\delta_{\mathrm{rk}}, \\
\int_{0}^{L} \operatorname{EI} \phi_{r}(x) \frac{\mathrm{d}^{4} \phi_{k}(x)}{\mathrm{d} x^{4}} \mathrm{~d} x=\omega_{r}^{2} \delta_{\mathrm{rk}} .
\end{gathered}
$$

From equations (27) and (28), we obtain

$$
\begin{aligned}
V_{j 1} & =-\frac{i \omega \vartheta}{i \omega C_{p, j 1}+Y_{j 1}} \sum_{r=1}^{N} \Delta \phi_{r, j 1}{ }^{\prime} \bar{\eta}, \\
V_{j 2} & =-\frac{i \omega \vartheta}{i \omega C_{p, j 2}+Y_{j 2}} \sum_{r=1}^{N} \Delta \phi_{r, j 2}{ }^{\prime} \bar{\eta},
\end{aligned}
$$

where $V_{j 1}$ and $V_{j 2}$ are the voltage amplitudes of the two subcells. Substituting equations (32) and (33) into equation (26), multiplying by $\phi_{n}(x)$, and integrating over the beam length from 0 to $L$ and then using the orthogonal conditions, the discretized governing equation can be obtained

$$
\begin{gathered}
\left(\omega_{r}^{2}-\omega^{2}\right) \bar{\eta}_{r}+\frac{i \omega \vartheta^{2}}{i \omega C_{\mathrm{p}, j 1}+Y_{j 1}} \sum_{j=1}^{S} \Delta \phi_{r, j 1} \sum_{k=1}^{N} \Delta \phi_{k, j 1}{ }^{\prime} \bar{\eta}_{k} \\
+\frac{i \omega \vartheta^{2}}{i \omega C_{\mathrm{p}, j 2}+Y_{j 2}} \sum_{j=1}^{S} \Delta \phi_{r, j 2} \sum_{k=1}^{N} \Delta \phi_{k, j 2}{ }^{\prime} \bar{\eta}_{k}=Q_{r},
\end{gathered}
$$

where

$$
Q_{r}=\bar{w}_{b} \omega^{2} \frac{1}{l} \int_{0}^{l} \phi_{k}(x) \mathrm{d} x
$$

By solving equation (34), the values of generalized coordinates can be calculated; substituting them into equation (30), the relative deflection amplitude can be obtained. The frequency response functions of the metamaterial beam are defined and calculated as

$$
T=\frac{\left|W_{\mathrm{abs}}(L)\right|}{\left|W_{b}\right|} .
$$

In summary, we proposed two methods to treat the dispersions: the transfer matrix method based on the state vectors and the continuity conditions between the two subcells and the units and the discretization method based on the vibrational point of view. The band structures of the proposed periodic structures will be derived by the transfer matrix method and the discretization method will be applied as numerical validation. Hence, equations (25) and (36) will be used in the following sections.

\section{Dispersion Relations of the Adaptive Metamaterial Beam}

In this section, the complex band structures of the metamaterial beam with various values of the shunt circuit elements containing resistance and inductance will be discussed based on the transfer matrix method. The parameters used in this study have been listed in Table 1.

3.1. Bragg Bandgap in Pure Resistance Circuit. In a pure resistance circuit, there are only the resistance elements and the inherent capacitance of the piezoelectric layers in the circuits which cannot generate resonant frequency. It is shown when the resistance values of the two circuits differ greatly and can be approximated as an open circuit and short circuit, the structure can only generate Bragg bandgap, and the band characteristics are most obvious. Figure 2 shows the first two-order Bragg bandgaps by $R_{1}=10^{-6} \Omega$ and $R_{2}=10^{6} \Omega$. The Bragg bandgaps in pure inductance circuit mainly reflected in the first- and second-order bandgaps and the bandwidths are generally narrow.

The impedance here rests with the resistance values of the external circuit. It has little effect on the band frequency region and bandwidth of the Bragg bandgap except the amplitude of the propagation constant.

3.2. Dispersion Relations in Pure Inductance Circuit. The band structure caused by the inductance circuit is different from that caused by the resistance circuit. In a pure inductance circuit, the circuit contains an inductance element, which forms an LC oscillation circuit with the inherent capacitance of the piezoelectric layer. The impedances mentioned in equations (2) and (3) are $i \omega_{e 1} L_{1}$ and $i \omega_{e 2} L_{2}$, where $\omega_{e 1}$ and $\omega_{e 2}$ are the oscillation frequencies of the two circuits. Due to the existence of the oscillation frequency, the metamaterial beam containing a pure inductance circuit can produce both locally resonant bandgap and Bragg bandgap. Moreover, the resonant bandgap frequencies are generally lower than Bragg bandgap frequencies. 
TABLe 1: Parameters of the metamaterial beam.

\begin{tabular}{lcc}
\hline Parameter & Symbol & Value \\
\hline Length of the unit cell & $2 l$ & $50 \mathrm{~mm}$ \\
Thickness of the host beam & $h_{\mathrm{s}}$ & $5 \mathrm{~mm}$ \\
Thickness of the piezoelectric layer & $h_{\mathrm{p}}$ & $0.5 \mathrm{~mm}$ \\
Width of the metamaterial beam & $b$ & $20 \mathrm{~mm}$ \\
Young's modulus of host beam & $E_{\mathrm{s}}$ & $69 \mathrm{GPa}$ \\
Density of the host beam & $\rho_{\mathrm{s}}$ & $2700 \mathrm{kgm}^{-3}$ \\
Young's modulus of the piezoelectric layer & $E_{\mathrm{p}}$ & $60.6 \mathrm{GPa}^{-3}$ \\
Density of the piezoelectric layer & $\rho_{\mathrm{p}}$ & $7500 \mathrm{kgm}^{-3}$ \\
Piezoelectric strain constant & $d_{31}$ & $-2.74 e^{-10} \mathrm{CN}^{-1}$ \\
Permittivity component & $\varepsilon_{33}^{S}$ & $2.56 e^{-10} \mathrm{Fm}^{-1}$ \\
\hline
\end{tabular}

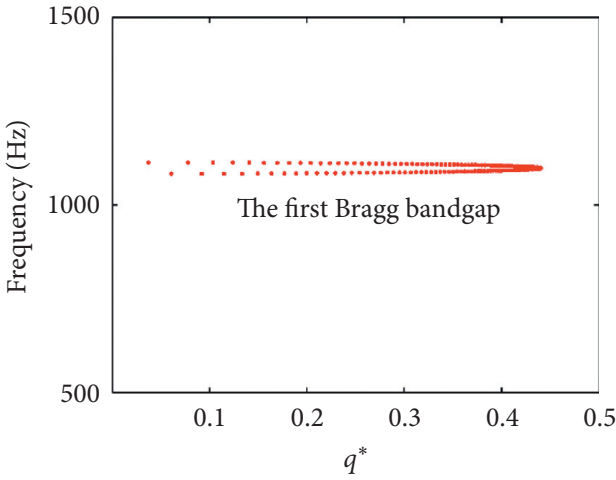

(a)

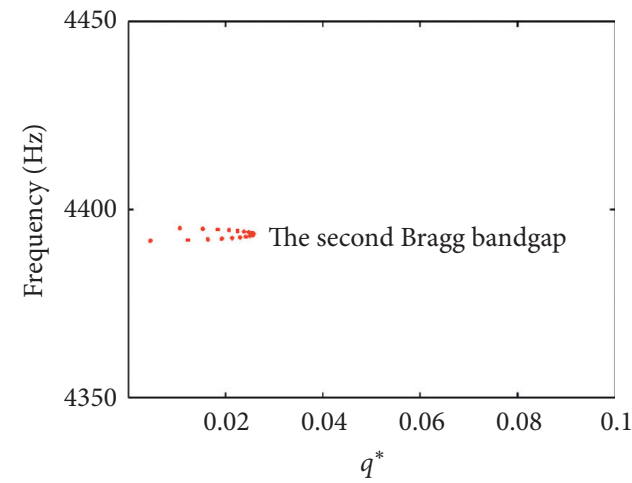

(b)

Figure 2: The band structure of the electromechanical metamaterial beam with $R_{1}=10^{-6} \Omega$ and $R_{2}=10^{6} \Omega$ : (a) the first Bragg bandgap and (b) the second Bragg bandgap.

3.2.1. Bragg Bandgap. In a pure inductance circuit, the addition of inductance will also cause the impedance mismatch between the two subcells. In addition, the oscillation frequency generated by the circuits will also affect the Bragg bandgap and the locally resonant bandgap.

Similar to the case of the pure resistance circuit, the Bragg bandgap appearing under the condition of the shunt circuit connected to $L_{1}$ is equivalent to an open circuit, while the shunt circuit connected to $L_{2}$ is equivalent to a short circuit. Figures 2(a) and 2(b) show the same phenomenon that the first two Bragg bandgaps with $L_{1}$ approach infinity and $L_{2}$ approach zero. The locally resonant bandgap generated by $L_{1}$ is infinitesimal which can be ignored and that generated by $L_{2}$ is infinite, and the system is mainly manifested as Bragg type. Moreover, the imaginary part value of the propagation constant is also relatively small.

3.2.2. Locally Resonant Bandgap. In general, short and open circuits are not considered, the resonant frequency generated by the inductance circuit is limited, and the impedance difference between the subcells is not significant. In this case, the electromechanical metamaterial beam mainly exhibits resonant bandgap in a pure inductance circuit. Next, the effect of the inductance value on locally resonant bandgap is discussed.

Considering the particular case $L_{1}=L_{2}$, the impendence and the natural frequency of the two subcells are exactly the same, and the model can be reduced to traditional electromechanical metamaterials. The corresponding local resonant bandgap is shown in Figure 3. It is noted that the bandwidth is narrower and the frequency range is lower than that of the Bragg bandgap. The bandwidth here seems to be relatively narrow because the electromechanical coupling of PZT-5H piezoelectric material is relatively weak. If the piezoelectric material is replaced by that with higher electromechanical coupling coefficient, the bandwidth here will be increased.

Figure 4 shows the band structures of the electromechanical metamaterials beam for different values of the inductance. It can be observed that two separate local resonant bandgaps appear due to the inequality of the inductance values. As we all know, the larger the inductance, the smaller the natural frequency of the corresponding circuit. So the resonant bandgap caused by $L_{1}$ has a higher frequency and a wider frequency region than that of $L_{2}$.

In order to predict the influence of inductance on locally resonant bandgap, the assumption of $L_{1}=10 \mathrm{H}$ is given and the bandwidth with respect to various values of $L_{2}$ is discussed. According to Figure 5(a), the position and width of the bandgap generated by subcell 1 are determined with bound frequencies $\omega$ from $113.46 \mathrm{~Hz}$ to $117.03 \mathrm{~Hz}$, and the bandwidth and frequency range are generated by subcell 2 decrease as $L_{2}$ increases. When $L_{2}$ increases to a certain value, the local resonant bandgap generated by subcell 1 and the bandgap generated by subcell 2 will be infinitely close to 


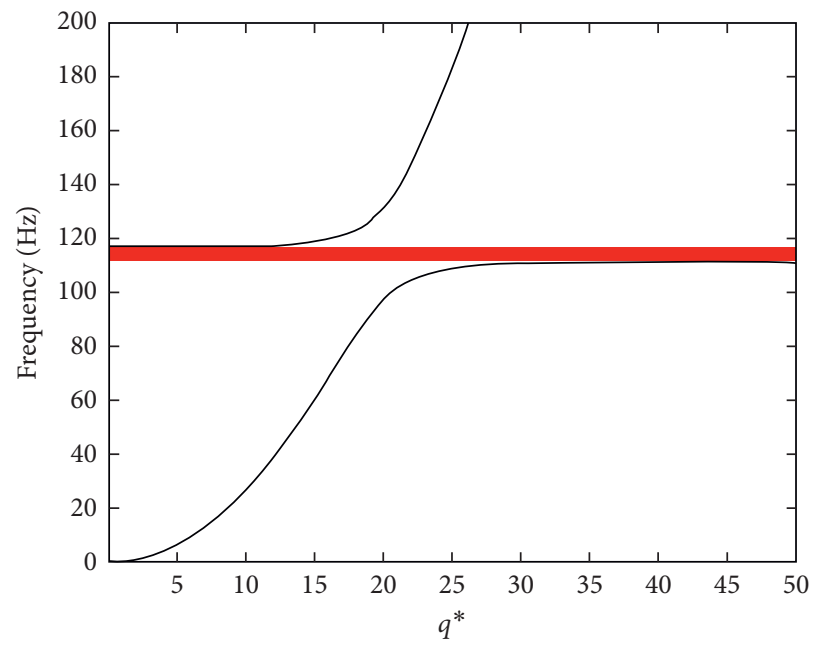

Bandgap $L_{1}=L_{2}=10 \mathrm{H}$

Figure 3: Band structures of the metamaterial beam in pure inductance circuit when $l_{1}=l_{2}=25 \mathrm{~mm}$ and $L_{1}=L_{2}=10 \mathrm{H}$.

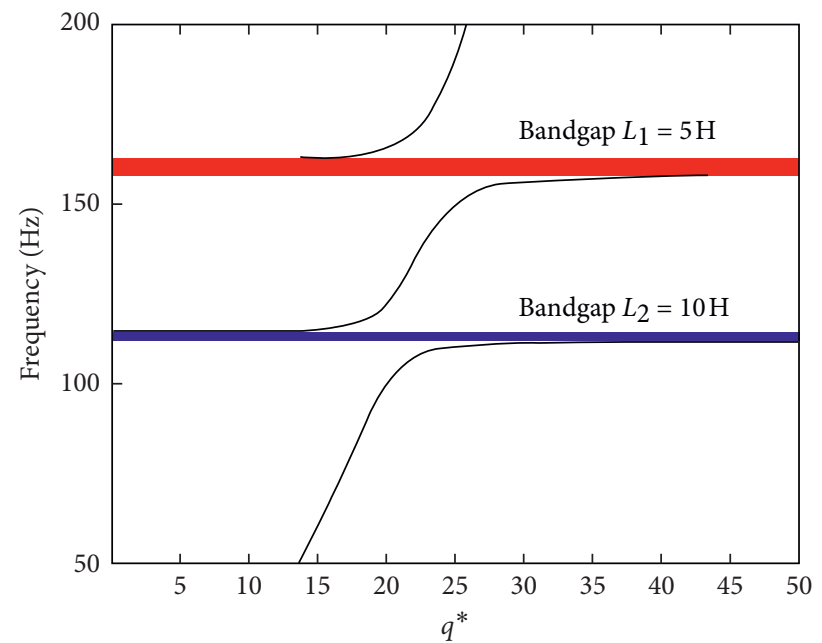

Figure 4: The locally resonant band structure of the metamaterial beam in pure inductance circuit when $L_{1}=5 \mathrm{H}$ and $L_{2}=10 \mathrm{H}$.

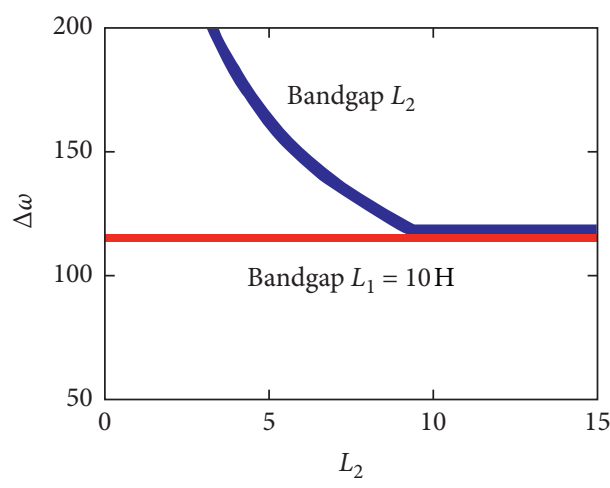

(a)

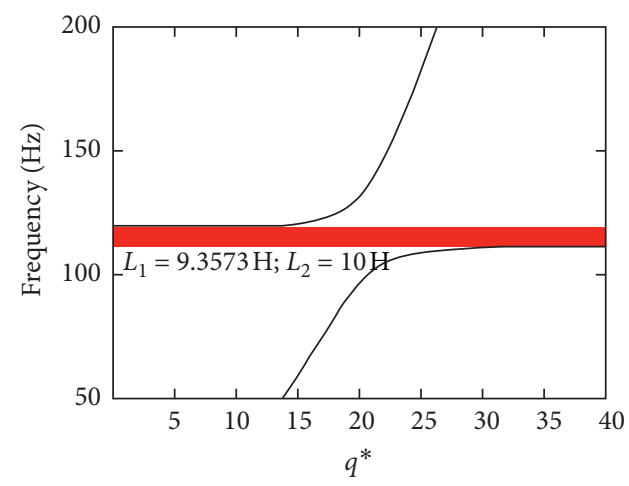

(b)

FIGURE 5: The locally resonant band structure of the metamaterial beam in pure inductance circuit with (a) $L_{1}=10 \mathrm{H}$ and different $L_{2}$ and (b) $L_{1}=9.3573 \mathrm{H}$ and $L_{2}=10 \mathrm{H}$. 
each other. Therefore, determining appropriate inductance or effective modulus can be employed to achieve a single lower and wider bandgap. As shown in Figure 5(b), it is theoretically possible to perfectly combine the two bandgaps by setting $L_{2}=10 \mathrm{H}$ and $L_{1}=9.3573 \mathrm{H}$, and the frequency ranges are exactly adjacent, creating a single and wider bandgap. For the structure proposed in this paper, wider and lower bandgap structures can be obtained. In general, when the structure contains multiple subunits, the bandgap will be extremely wide and low.

3.3. The Influence of length Ratio on Dispersion Relations. In this section, the effect of the length of the two subcells on band structure will be discussed. Firstly, the coefficient $\eta$ is introduced which can be written as $\eta=l_{1} /\left(l_{1}+l_{2}\right)$, where $l_{1}$ and $l_{2}$ are the length of the subcell 1 and subcell 2 .

3.3.1. Bragg Bandgap in Pure Inductance Circuit. Based on the results given in Sections 3.1 and 3.2, the Bragg bandgap is most obvious when the circuits connected to the two subcells are open and short, respectively. Here, the resistance values of the two subcells circuits are kept at constant $R_{1}=10^{6} \Omega$ and $R_{2}=10^{-6} \Omega$, respectively. Figure 6 shows the Bragg bandgaps with various $\eta$. It should be noted that, with the increase of the length ratio $\eta$, the location of the first Bragg bandgap moves toward a higher frequency and the width of the Bragg bandgap varies to wider. The capacitance of the piezoelectric material described by equations (36) and (37) affected by the length of the two subcells can change the impendence and Bragg bandgap. Coincidentally, the phenomenon in the pure inductance circuits is similar to the pure resistance circuits.

\subsubsection{Locally Resonant Bandgap in Pure Inductance Circuit.} The capacitance of the two subcells is affected by the length. For the given inductance $L_{1}=L_{2}=10 \mathrm{H}$, Figure 7 shows the locally resonant bandgaps of the electromechanical metamaterial beam for various length ratio $\eta$. Comparing the four plots, it is found that the bandgap generated by subcell 1 with a higher frequency region will move toward lower frequency and the width will vary to wider with the increase of $\eta$. On the contrary, the bandgap generated by subcell 2 with lower frequency region will move toward a higher frequency and the width will vary to narrower. In addition, the locations of the two bandgaps are getting closer to each other. The two bandgaps are coincident consistent with the traditional piezoelectric metamaterials as shown in Figure 7(d) when $\eta=0.5$.

The bandwidth and frequency range of the locally resonant bandgap mainly depend on the value of $\eta$ when the inductance value is constant. Next, the relationship between the bandwidth of two locally resonant bandgaps and $\eta$ is analyzed. The bandwidth of the two subcells versus the length ratio $\eta$ is depicted in Figure 8(a). It can be observed that the bandgap generated by subcell 1 is shifted from highfrequency range to a lower one as $\eta$ increase, and the bandwidth becomes wider. In contrast, the position and width of the bandgap generated by subcell 2 are opposite to the bandgap generated by subcell 1 . It also can be seen that

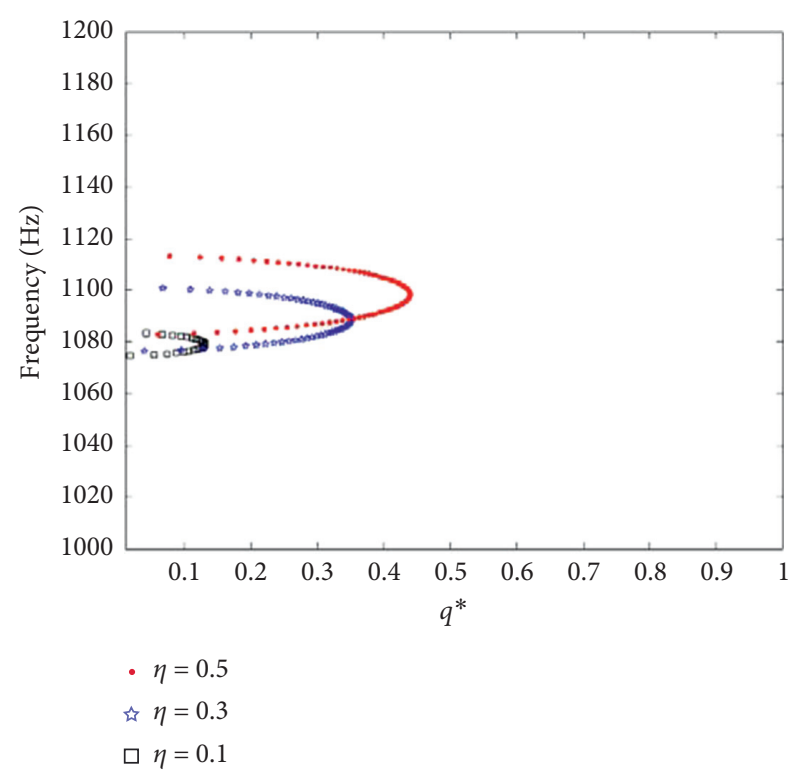

FIgURE 6: The first Bragg band structures in pure resistance and pure inductance circuit with $\eta=0.1, \eta=0.3$, and $\eta=0.5$.

there are two values near both sides of $\eta=0.5$ which can widen the bandgap. As shown in Figure 8(b), there are similar phenomena to $L_{1}=L_{2}=10 \mathrm{H}$, but no symmetry due to the unequal of the inductance values. In addition, there are two length ratios near the location where the two frequency ranges overlap which can tune the two bandgaps to be adjacent, creating a single, wider bandgap.

According to Sugino et al. [13], the starting and ending frequency of the local resonant bandgap with electromechanical resonators can be given by

$$
\begin{aligned}
& \omega_{\text {starting }}=\frac{\omega_{e}}{\sqrt{1+\alpha}}, \\
& \omega_{\text {ending }}=\omega_{e},
\end{aligned}
$$

where $\alpha$ is the electromechanical coupling coefficient, which measures how strongly coupled the piezoelectric material is to the host beam. Then it can be written as

$$
\alpha=\frac{e_{31}^{2} \mathrm{bh}_{p}\left(h_{s}+h_{p}\right)^{2}}{2 \mathrm{EI} \varepsilon_{33}^{S}} .
$$

Based on equation (38), one can easily find that the electromechanical coupling coefficient is affected by the geometric and structural parameters of the metamaterial beam. To obtain a wider locally resonant bandgap, the frequencies of the electromechanical resonators should be selected as $\omega_{s 1}=\omega_{e 2}$, and the bandgap can be widened at $\eta=0.328654$, where the ending frequency of the bandgap generated by subcell 2 and the starting frequency of the bandgap generated by subcell 1 coincide as seen in Figure 9 .

\section{Numerical Results}

The frequency response functions in an elastic metamaterial beam can examine the wave properties effectively. However, 


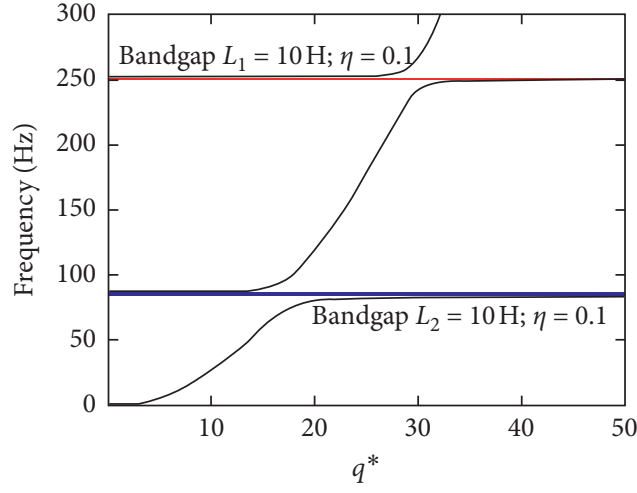

(a)

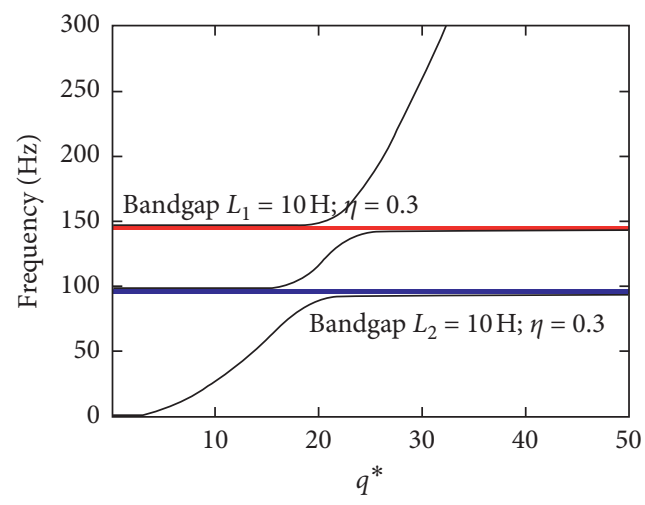

(c)

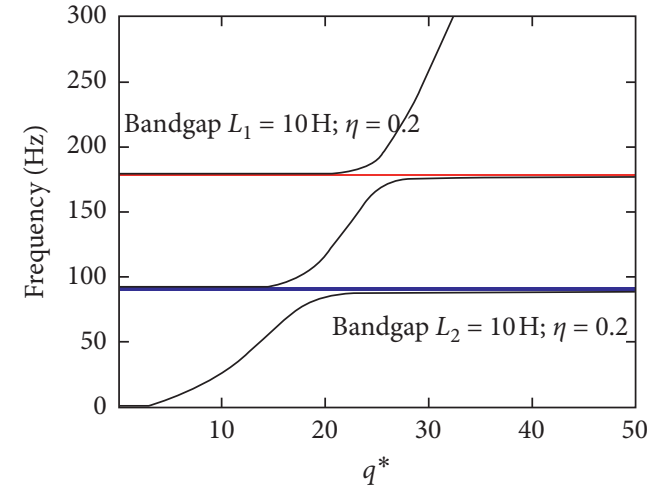

(b)

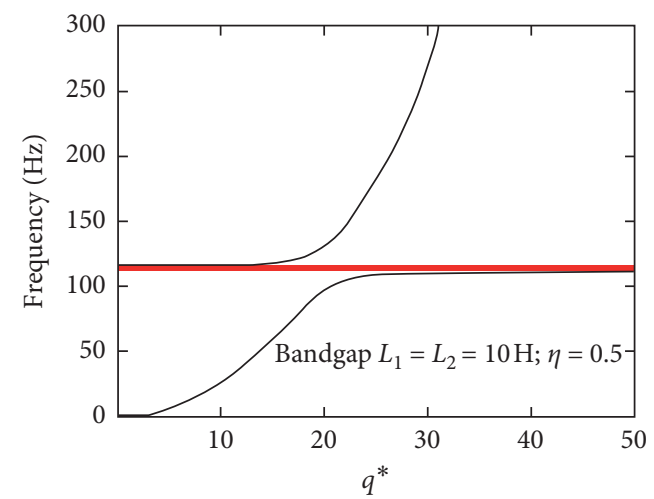

(d)

Figure 7: The locally resonant band structures of the metamaterial beam in pure inductance circuit, (a) $\eta=0.1$, (b) $\eta=0.2$, (c) $\eta=0.3$, and (d) $\eta=0.5$.

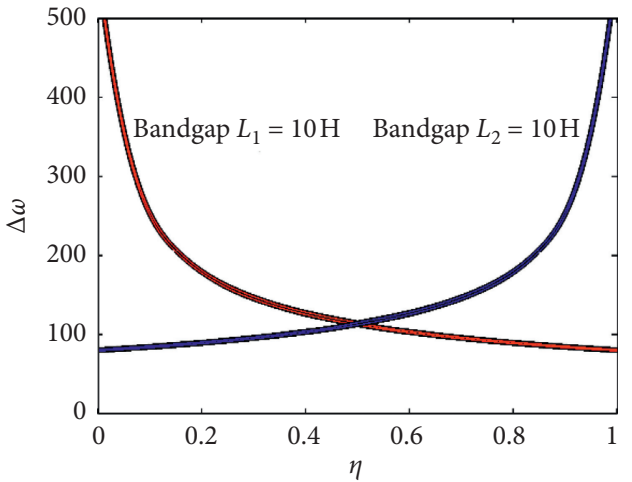

(a)

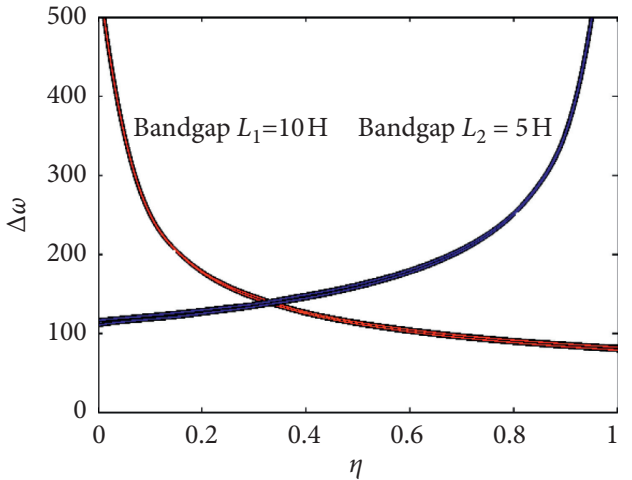

(b)

FIGURE 8: Bandwidth of the two subunits in pure inductance circuit with various length ratios with (a) $L_{1}=L_{2}=10 \mathrm{H}$ and (b) $L_{1}=10 \mathrm{H}$ and $L_{2}=5 \mathrm{H}$.

for a finite length of the electromechanical metamaterial beam, the number of unit cells and the order of truncation will always affect the efficiency of wave attenuation. In order to explore the influence of various circuit elements on wavepropagation characteristics, the numerical simulations of wave attenuation with twenty unit cells and eight orders truncation are conducted based on equation (36). In the calculations, the geometric and structural parameters are also the same as listed in Table 1, and the length of metamaterial beam and each subcell is $L=1 \mathrm{~m}$ and $l=25 \mathrm{~mm}$.
4.1. Bragg Bandgap in Open-Short Circuit. In pure resistance circuit or pure inductance circuit, the governing equations (27) and (28) for shunting circuits of the two subcells have the same form except the admittances $Y_{j}$, where $1 / R_{1}$ and $1 / R_{2}$ are for resistance circuit and $1 / i \omega_{e 1} L_{1}$ and $1 / i \omega_{e 2} L_{2}$ are for inductance circuit. As can be seen from Section 3.2, the Bragg bandgaps in the pure resistance circuit or the pure inductance circuit are uniform.

The frequency responses of the electromechanical metamaterial beam for open-short circuits are shown in Figure 10. It 


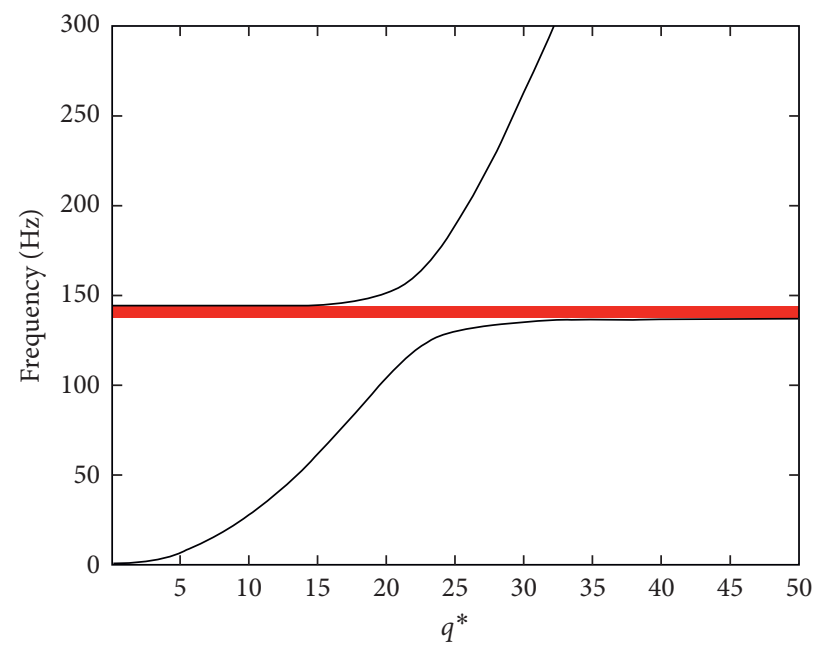

Bandgap $\eta-0.328654$

Figure 9: The locally resonant band structures of the metamaterial beam in pure inductance circuit with $\eta=0.328654$.

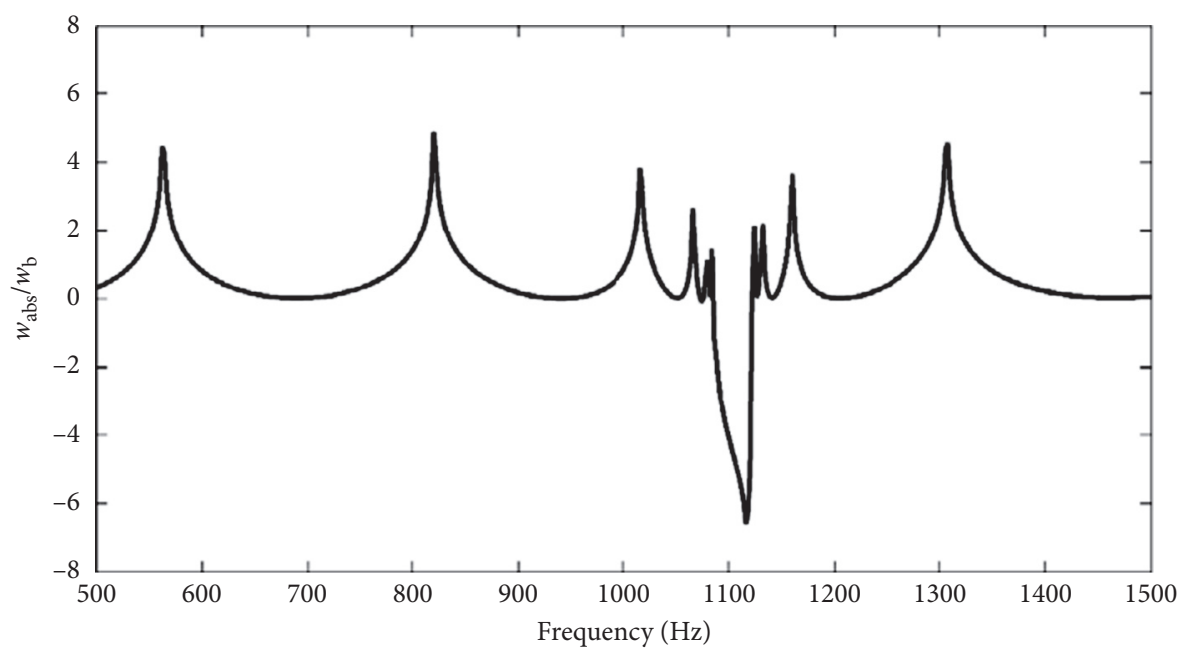

FIGURE 10: Response of the metamaterial beam in open-short circuits.

shows that the first Bragg bandgap shown in Figure 2 and the current frequency responses are in good agreement with each other.

\subsection{Locally Resonant Bandgap in Pure Inductance Circuit.} Figure 11(a) shows the frequency responses of the traditional metamaterial beam when the piezoelectric layers are connected with pure inductance circuits by setting $L_{1}=L_{2}=10 \mathrm{H}$. Here, geometric and structural parameters are the same as those used in Figure 10. As illustrated in Figure 11(a), a single narrow bandgap is observed at frequencies between $112.71 \mathrm{~Hz}$ and $117.10 \mathrm{~Hz}$. This transmittance dip and the bandgap frequencies predicted by the analytical dispersion relations shown in Section 3 are in good agreement with each other. Figure 11(b) shows the frequency responses of the electromechanical metamaterial beam with $L_{1}=10 \mathrm{H}$ and $L_{2}=5 \mathrm{H}$, and it is clear from the figure that there are two narrow separate bandgaps whose frequencies are between $113.22 \mathrm{~Hz}$ and $117.10 \mathrm{~Hz}$ and the other frequencies are between $159.52 \mathrm{~Hz}$ and $165.81 \mathrm{~Hz}$. In order to achieve the desired performance demonstrated in Figure 5(b), the inductance of the subcell should be modified slightly. Specifically, for the pure inductance circuit, $L_{2}$ is selected as $9.3573 \mathrm{H}$. In this case, the ending frequency of the bandgap generated by subcell 2 and the starting frequency of the bandgap generated by subcell 1 coincide. As shown in Figure 11(c), the frequency responses of the electromechanical metamaterial beam with $L_{1}=10 \mathrm{H}$ and $L_{2}=9.3573 \mathrm{H}$ demonstrate a single and wider bandgap from $113.11 \mathrm{~Hz}$ to $121.79 \mathrm{~Hz}$. It should be noted that a transmission peak exists inside the widen bandgap, which is caused by the coupling between the two circuits of the adjacent subcells. In the future, geometric optimizations are still in demanding in attenuation performance.

As Bragg bandgap can only be obtained when the circuits of the two adjacent resonators are approximate to short circuit and open circuit, the existing formula (i.e., equation (37)) cannot predict the Bragg bandgap. Table 2 compares the predictions of the locally resonant bandgap from band 


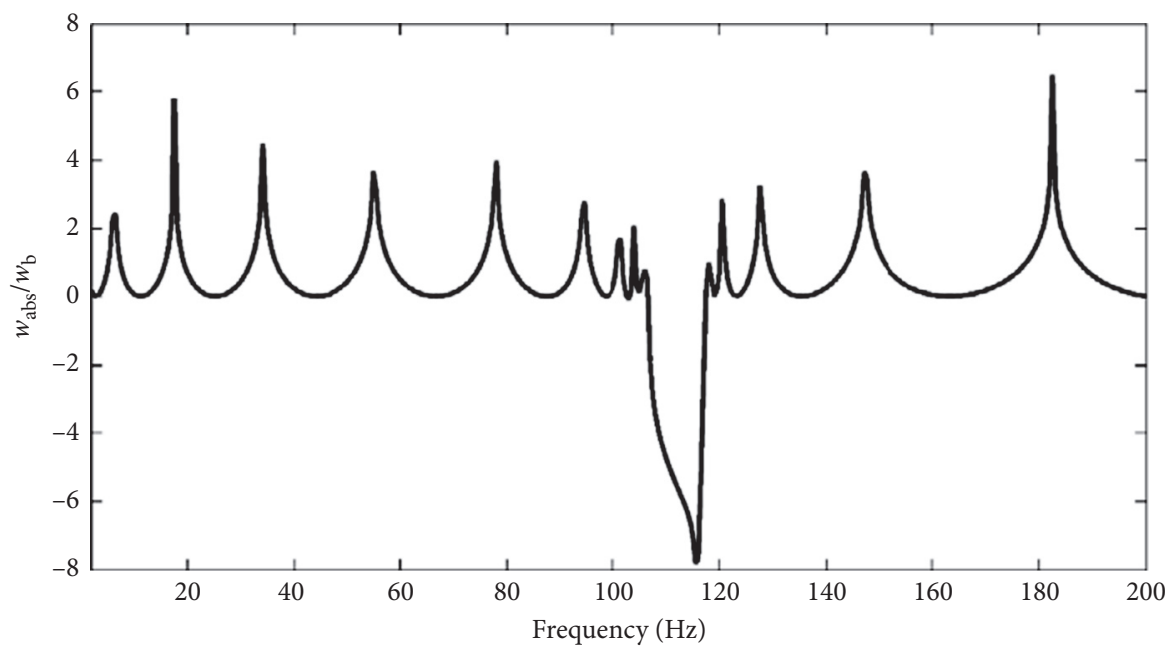

$L_{1}=10 \mathrm{H} ; L_{2}=10 \mathrm{H}$

(a)

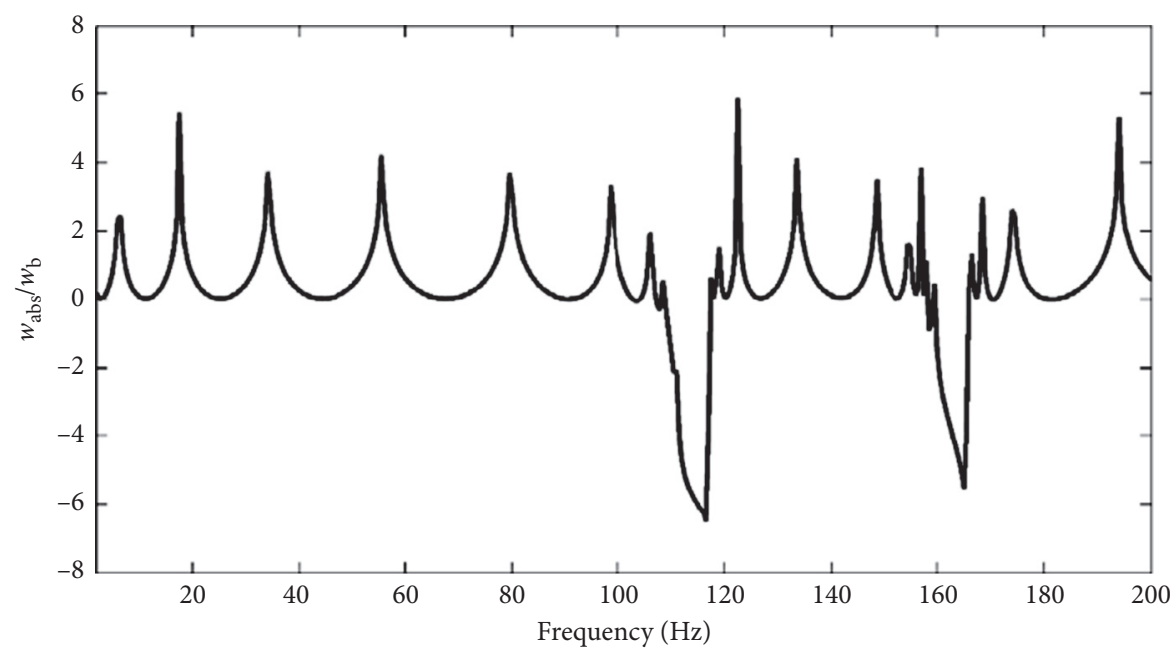

$L_{1}=10 \mathrm{H} ; L_{2}=5 \mathrm{H}$

(b)

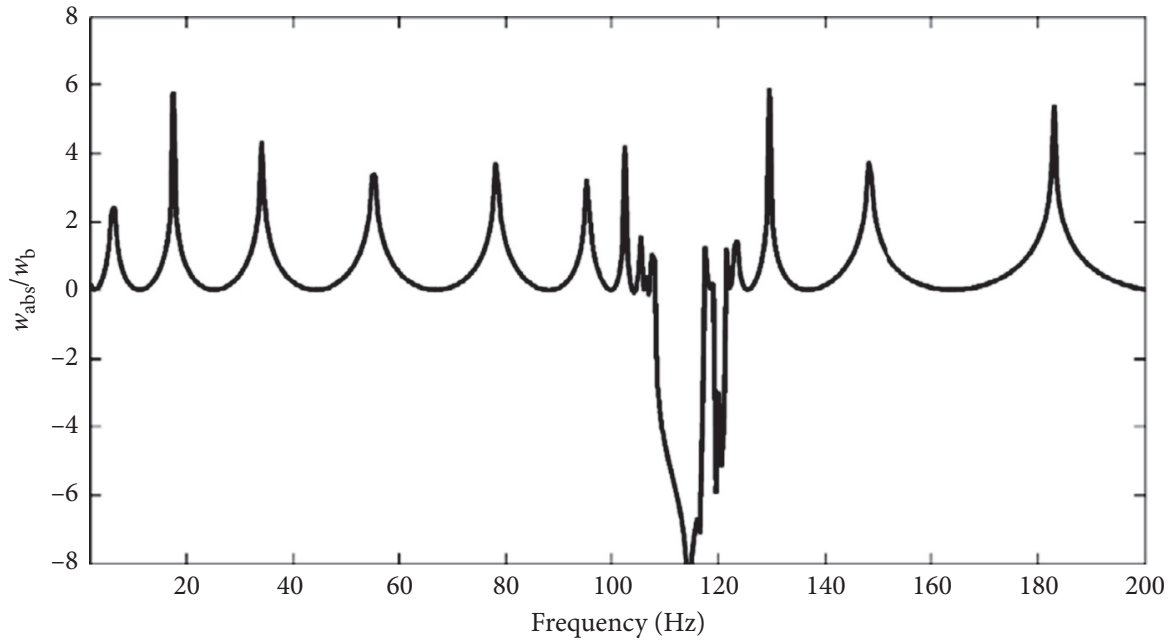

$L_{1}=10 \mathrm{H} ; L_{2}=9.3573 \mathrm{H}$

(c)

FIGURE 11: Frequency response function of the electromechanical metamaterial beam: (a) $L_{1}=L_{2}=10 \mathrm{H}$; (b) $L_{1}=10 \mathrm{H}$ and $L_{2}=5 \mathrm{H}$; (c) $L_{1}=10 \mathrm{H}$ and $L_{2}=9.3573 \mathrm{H}$. 
TABLE 2: Comparisons of the bandgap prediction.

\begin{tabular}{lccc}
\hline Inductance $(\mathrm{H})$ & Band analysis $(\mathrm{Hz})$ & Transmittance $(\mathrm{Hz})$ & Existing formula $(\mathrm{Hz})$ \\
\hline$L_{1}=10$ and $L_{2}=10$ & $113.29-117.05$ & $112.71-117.10$ & $113.46-117.05$ \\
$L_{1}=10$ and $L_{2}=5$ & $113.26-117.05$ & $113.22-117.10$ & $113.46-117.05$ \\
$L_{1}=10$ and $L_{2}=9.3573$ & $160.39-165.54$ & $159.52-165.81$ & $160.46-165.53$ \\
\hline
\end{tabular}

structure analysis, transmittance, and the existing formula (i.e., equation (37)). The parameters for calculating the results of Table 2 can be obtained from Table 1 . The length ratio is taken as 0.5 , and the electromechanical coupling coefficient of the two subcells $\alpha$ is 0.0642 .

It can be seen from Table 2 that the starting frequency of the bandgap obtained by using the band structure analysis is basically the same as that obtained by the existing formula (i.e., equation (37)), and the ending frequency of the bandgap obtained by using the band structure analysis is slightly smaller than that obtained by using the existing formula. Due to the difference in the computational accuracy between the band structure analysis and the existing formula, the bandgap obtained is slightly different. In addition, when considering twenty unit cells and eighth order truncations, the transmittance obtained by equation (36) has a high accuracy, which can be used to verify well in the band structure. In general, the band structure analysis, transmittance, and the existing formula (i.e., equation (37)) are in good agreement with each other in bandgap prediction.

\section{Conclusions}

In analogy with the mechanical metamaterial, electromechanical metamaterial made from elastic substrates with piezoelectric layers shunted to shunting circuits also exhibits bandgaps. However, the bandgap formation in electromechanical metamaterial is associated with a frequency-dependent modal stiffness and the frequency-dependent modal mass in the mechanical metamaterial. Here, an adaptive metamaterial beam with a couple of electromechanical resonators in each unit cell is proposed to open multiple locally resonant bandgaps or Bragg bandgaps. We presented a detailed analytical and numerical investigation of the metamaterial beam with electromechanical resonators. The numerical results show that the Bragg bandgap of the system is the most obvious when the pure resistance circuit of two subcells in a unit can be approximated as open circuit and short circuit, respectively. Similarly, when the pure inductance circuits of the two subcells are approximate to open and short circuit respectively, the system also presents mainly Bragg bandgap characteristics. Otherwise, multiple separate locally resonant bandgaps can be created. The frequency responses as numerical validation are in good agreement with the analytical bandgaps. It is concluded that the length ratio $\eta$ can also affect the band structures dramatically by varying the capacitance of the subcell. Further, the local resonant bandgap can be broadened, creating a single, wider local resonant bandgap, by adding two resonators to the unit cell with the resonant frequencies of the shunting circuits satisfying a proper ratio. In general, when the structure contains multiple subunits, the bandgap will be extremely wide and low. The system proposed in this paper opens up important perspectives for the development of adaptive vibration or wave-attenuation devices for broadband and low-frequency applications.

\section{Data Availability}

All the data used in the study are available. Previously reported data were used to support this study and are available. These prior studies are cited at relevant places within the text as references.

\section{Conflicts of Interest}

The authors declare that there are no conflicts of interest regarding the publication of this paper.

\section{Acknowledgments}

The authors gratefully acknowledge the support from the National Natural Science Foundation of China (Grant no. 11672007) and Beijing Natural Science Foundation (Grant no. 3172003$)$.

\section{References}

[1] M. I. Hussein, M. J. Leamy, and M. Ruzzene, "Dynamics of phononic materials and structures: historical origins, recent progress, and future outlook," Applied Mechanics Reviews, vol. 66, no. 4, Article ID 040802, 2014.

[2] J. Zhou, L. Dou, K. Wang, D. Xu, and H. Ouyang, "A nonlinear resonator with inertial amplification for very low-frequency flexural wave attenuations in beams," Nonlinear Dynamics, vol. 96, no. 1, pp. 647-665, 2019.

[3] Z. S. Chen, J. He, and G. Wang, "Vibration bandgaps of piezoelectric metamaterial plate with local resonators for vibration energy harvesting," Shock and Vibration, vol. 2019, Article ID 1397123, 15 pages, 2019.

[4] Z. Liu, X. X. Zhang, Y. W. Mao et al., "Locally resonant sonic materials," Science, vol. 289, no. 5485, pp. 1734-1736, 2000.

[5] E. J. P. Miranda and J. M. C. Dos Santos, "Flexural wave bandgaps in multi-resonator elastic metamaterial Timoshenko beams," Wave Motion, vol. 91, Article ID 102391, 2019.

[6] P. F. Pai, H. Peng, and S. Jiang, "Acoustic metamaterial beams based on multi-frequency vibration absorbers," International Journal of Mechanical Sciences, vol. 79, pp. 195-205, 2014.

[7] H. Peng, P. Frank Pai, and H. Deng, "Acoustic multi-stopband metamaterial plates design for broadband elastic wave absorption and vibration suppression," International Journal of Mechanical Sciences, vol. 103, pp. 104-114, 2015.

[8] C. Sugino, S. Leadenham, M. Ruzzene, and A. Erturk, "On the mechanism of bandgap formation in locally resonant finite 
elastic metamaterials," Journal of Applied Physics, vol. 120, no. 13, Article ID 134501, 2016.

[9] J. X. Zhou, K. Wang, D. L. Xu, and H. J. Ouyang, "Local resonator with high-static-low-dynamic stiffness for lowering bandgaps of flexural wave in beams," Journal of Applied Physics, vol. 121, Article ID 044902, 2017.

[10] J. Zhou, K. Wang, D. Xu, and H. Ouyang, "Multi-low-frequency flexural wave attenuation in Euler-Bernoulli beams using local resonators containing negative-stiffness mechanisms," Physics Letters A, vol. 381, no. 37, pp. 3141-3148, 2017.

[11] R. L. Forward, "Electronic damping of vibrations in optical structures," Applied Optics, vol. 18, no. 5, pp. 690-697, 1979.

[12] N. W. Hagood and A. von Flotow, "Damping of structural vibrations with piezoelectric materials and passive electrical networks," Journal of Sound and Vibration, vol. 146, no. 2, pp. 243-268, 1991.

[13] C. Sugino, S. Leadenham, M. Ruzzene, and A. Erturk, "An investigation of electroelastic bandgap formation in locally resonant piezoelectric metastructures," Smart Materials and Structures, vol. 26, no. 5, Article ID 055029, 2017.

[14] C. Sugino, Y. Xia, S. Leadenham, M. Ruzzene, and A. Erturk, "A general theory for bandgap estimation in locally resonant metastructures," Journal of Sound and Vibration, vol. 406, pp. 104-123, 2017.

[15] J. Xu, R. Yan, and J. Tang, "Broadening bandgap width of piezoelectric metamaterial by introducing cavity," Applied Sciences, vol. 8, no. 9, p. 1606, 2018.

[16] Y. Y. Chen, G. K. Hu, and G. L. Huang, "An adaptive metamaterial beam with hybrid shunting circuits for extremely broadband control of flexural waves," Smart Materials and Structures, vol. 25, no. 10, Article ID 105036, 2016.

[17] Y. H. Lia, Y. Y. Chen, G. L. Huang, and X. M. Zhou, "Broadband low-frequency sound isolation by lightweight adaptive metamaterials," Journal of Applied Physics, vol. 123, Article ID 091705, 2018.

[18] Y. Chen, G. Hu, and G. Huang, "A hybrid elastic metamaterial with negative mass density and tunable bending stiffness," Journal of the Mechanics and Physics of Solids, vol. 105, pp. 179-198, 2017.

[19] C. Sugino, M. Ruzzene, and A. Erturk, "Merging mechanical and electromechanical bandgaps in locally resonant metamaterials and metastructures," Journal of the Mechanics and Physics of Solids, vol. 116, pp. 323-333, 2018.

[20] H. Zhang, J. Wen, Y. Xiao, G. Wang, and X. Wen, "Sound transmission loss of metamaterial thin plates with periodic subwavelength arrays of shunted piezoelectric patches," Journal of Sound and Vibration, vol. 343, pp. 104-120, 2015.

[21] N. Kherraz, L. Haumesser, F. Levassort, P. Benard, and B. Morvan, "Hybridization bandgap induced by an electrical resonance in piezoelectric metamaterial plates," Journal of Applied Physics, vol. 123, Article ID 094901, 2018.

[22] C. Sugino, M. Ruzzene, and A. Erturk, "An analytical framework for Kirchhoff plate-type locally resonant piezoelectric metastructures," in Proceedings of the Active and Passive Smart Structures and Integrated Systems XIII, A. Erturk, Ed., Denver, CO, USA, March 2019.

[23] F. M. Li and C. Z. Zhang, "Active localization of wave propagation in elastic beams using periodic placement of piezoelectric actuator/sensor pairs," Journal of Applied Physics, vol. 124, Article ID 085106, 2018.

[24] F. Li, C. Zhang, and C. Liu, "Active tuning of vibration and wave propagation in elastic beams with periodically placed piezoelectric actuator/sensor pairs," Journal of Sound and Vibration, vol. 393, pp. 14-29, 2017.
[25] J.-S. Chen, W.-J. Su, Y. Cheng, W.-C. Li, and C.-Y. Lin, “A metamaterial structure capable of wave attenuation and concurrent energy harvesting," Journal of Intelligent Material Systems and Structures, vol. 30, no. 20, pp. 2973-2981, 2019.

[26] M. Hajhosseini and M. Rafeeyan, "Modeling and analysis of piezoelectric beam with periodically variable cross-sections for vibration energy harvesting," Applied Mathematics and Mechanics, vol. 37, no. 8, pp. 1053-1066, 2016.

[27] G. B. Hu, L. H. Tang, and R. Das, "Internally coupled metamaterial beam for simultaneous vibration suppression and low frequency energy harvesting," Journal of Applied Physics, vol. 123, Article ID 055107, 2018.

[28] C. Sugino and A. Erturk, "Analysis of multifunctional piezoelectric metastructures for low-frequency bandgap formation and energy harvesting," Journal of Physics D: Applied Physics, vol. 51, no. 21, Article ID 215103, 2018.

[29] Y. Xiao, J. Wen, and X. Wen, "Broadband locally resonant beams containing multiple periodic arrays of attached resonators," Physics Letters A, vol. 376, no. 16, pp. 1384-1390, 2012.

[30] H.-Y. Chen, X.-Y. Mao, H. Ding, and L.-Q. Chen, "Elimination of multimode resonances of composite plate by inertial nonlinear energy sinks," Mechanical Systems and Signal Processing, vol. 135, Article ID 106383, 2020. 